\title{
No Syllogisms for the Numerical Syllogistic
}

\author{
Ian Pratt-Hartmann \\ School of Computer Science, \\ University of Manchester, \\ Manchester M13 9PL, U.K. \\ ipratt@cs.man.ac.uk
}

\begin{abstract}
The numerical syllogistic is the extension of the traditional syllogistic with numerical quantifiers of the forms at least $C$ and at most $C$. It is known that, for the traditional syllogistic, a finite collection of rules, similar in spirit to the classical syllogisms, constitutes a sound and complete proof-system. The question arises as to whether such a proof system exists for the numerical syllogistic. This paper answers that question in the negative: no finite collection of syllogism-like rules, broadly conceived, is sound and complete for the numerical syllogistic.
\end{abstract}

\section{Introduction}

The numerical syllogistic is the set of English sentences of the forms

$$
\begin{array}{ll}
\text { At least } C p \text { are } q & \text { At least } C p \text { are not } q \\
\text { At most } C p \text { are } q & \text { At most } C p \text { are not } q,
\end{array}
$$

where $p$ and $q$ are common (count) nouns, and $C$ is a (decimal) digit string representing a natural number in the usual way. We here ignore, and henceforth silently correct, details of English number-agreement and plural morphology, since these matters have no bearing on the ensuing discussion. The argument

$$
\begin{aligned}
& \text { At least } 13 \text { artists are beekeepers } \\
& \text { At most } 3 \text { beekeepers are not carpenters } \\
& \text { At most } 1 \text { carpenter is not a dentist } \\
& \text { At least } 9 \text { artists are dentists, }
\end{aligned}
$$

whose premises and conclusion all belong to the numerical syllogistic, is evidently valid: any circumstance in which all the premises are true is one in which the conclusion is true. For suppose the premises are true. Take any collection of thirteen artists who are beekeepers; since at most three of these are not carpenters, the remaining ten are; and since, of these ten, at most one is not a dentist, the remaining nine are.

The numerical syllogistic generalizes the traditional syllogistic, which, for our purposes, we may take to be the set of English sentences of the forms

Some $p$ are $q$

All $p$ are $q$
Some $p$ are not $q$

No $p$ are $q$. 
To see this, note that the sentence Some $p$ are $q$ may be equivalently written At least $1 p$ is a $q$; likewise, All $p$ are $q$ may be equivalently (if somewhat unidiomatically) written At most $0 p$ are not $q$; and so on. Since the standard system of syllogisms presented in Aristotle's Prior Analytics can be shown-with a few relatively minor adjustments - to license exactly the valid arguments in the traditional syllogistic [1-4], it is natural to ask whether a similar situation holds for the numerical syllogistic.

To understand what this question means more concretely, consider the rule

$$
\frac{\text { At most } C p \text { are not } q \quad \text { At least } D o \text { are } p}{\text { At least } E o \text { are } q}(0 \leq E \leq D-C),
$$

which we interpret as licensing an inference from any instances of the sentenceschemata above the line to the corresponding instance of the sentence schema below the line, subject to the side-condition $0 \leq E \leq D-C$. Clearly, this rule is valid: it never leads from true premises to a false conclusion. For suppose the premises are true. Take any collection of $D o$ which are $p$; since at most $C$ of them are not $q$, the remaining $D-C$ are. In fact, by chaining two instances of Rule (2) together, we can formally demonstrate the validity of Argument (1), thus:

\begin{tabular}{lll} 
At most 1 carpenter is not a dentist & $\begin{array}{l}\text { At most } 3 \text { beekeepers } \\
\text { are not carpenters }\end{array}$ & $\begin{array}{l}\text { At least } 13 \text { artists } \\
\text { are beekeepers }\end{array}$ \\
\cline { 2 - 3 } & At least 10 artists are carpenters \\
\hline
\end{tabular}

At least 9 artists are dentists.

Rule (2) might reasonably be regarded as a "numerical syllogism". Indeed, the traditional syllogism Darii is simply the special case obtained by putting $C=0$ and $D=E=1$ :

$$
\frac{\text { All } p \text { are } q \quad \text { Some } o \text { are } p}{\text { Some } o \text { are } q} .
$$

Thus, we are led to ask whether there exists a finite collection of such numerical syllogisms - broadly conceived - that licenses all (and only) the valid arguments in the numerical syllogistic? We show in the sequel that there is not.

Despite its obviousness as a generalization of the traditional syllogistic, the numerical syllogistic seems not to have attracted the attention of logicians before the Nineteenth Century. The first systematic investigation known to the author is that of de Morgan [5] (Ch. VIII), though this work was closely followed by treatments in Boole [6] (reprinted as [7], Sec. IV) and Jevons [8], (reprinted as [9], Part I, Sec. IV). For a historical overview of this episode in logic, see [10]. De Morgan presented a list of what he took to be the valid numerical syllogisms. Latterly, various other proof-systems have been proposed, also based on numerical generalizations of the traditional syllogisms. Good examples are those of Murphree [11,12], and of Hacker and Parry [13]. The negative results presented below apply to all of these systems. These same results constitute a strengthening (and simplification) of earlier observations made by the author in [14], Sec. 5 . 
At the same time, the present paper can be seen as a contribution-though perhaps something of a negative one - to an established tradition of attempts to provide logical calculi more or less closely modelled on aspects of natural languages. Examples of work in this tradition include Fitch's use of combinatory logic [15], Suppes' use of relation algebra [16], Purdy's 'natural logic' [17-19], and Fyodorov et al.'s inference calculus based on monotonicity features [20].

The plan of the paper is as follows. Section 2 presents the syntax and semantics of a formal language, $\mathcal{N}$, which faithfully reconstructs the numerical syllogistic, together with a natural extension of $\mathcal{N}$, which we denote $\mathcal{N}^{\dagger}$. Section 3 reconstructs - as liberally as possible - the notion of a numerical syllogism, and states the main result of this paper: that neither $\mathcal{N}$ nor $\mathcal{N}^{\dagger}$ admit a finite system of numerical syllogisms that licenses exactly the valid inferences. In stating this result, we pay particular attention to indirect proof and the rule of reductio ad absurdum. Section 4 proves the result.

\section{Syntax and semantics of $\mathcal{N}^{\dagger}$ and $\mathcal{N}$}

Fix a countably infinite set $\mathbf{P}$. We may assume $\mathbf{P}$ to contain all English common count-nouns such as man, animal etc. An atom is an element of $\mathbf{P}$; a literal is an expression of either of the forms $p$ or $\bar{p}$, where $p$ is an atom. A literal which is an atom is said to be positive; all other literals are said to be negative. If $l=\bar{p}$ is a negative literal, then we take $\bar{l}$ to denote the positive literal $p$. An $\mathcal{N}^{\dagger}$-formula is an expression of either of the forms $(\leq C)[l, m]$ or $(>C)[l, m]$, where $C$ is a decimal string representing a non-negative integer, and $l, m$ are literals. To avoid cumbersome circumlocutions, we henceforth ignore the distinction between natural numbers and the decimal strings representing them. An $\mathcal{N}$-formula is an $\mathcal{N}^{\dagger}$-formula at least one of whose literals is positive. We denote the set of $\mathcal{N}^{\dagger}$-formulas by $\mathcal{N}^{\dagger}$; and similarly for $\mathcal{N}$. A subset $P \subseteq \mathbf{P}$ is a signature. If $P$ is a signature, we denote by $\mathcal{N}^{\dagger}(P)$ the set of $\mathcal{N}^{\dagger}$-formulas involving no atoms other than those in $P$; and similarly for $\mathcal{N}(P)$.

We provide formal semantics for the language $\mathcal{N}^{\dagger}$ - and hence for $\mathcal{N}$-as follows. A structure $\mathfrak{A}$ is a pair $\left\langle A,\left\{p^{\mathfrak{A}}\right\}_{p \in \mathbf{P}}\right\rangle$, where $A$ is a non-empty set, and $p^{\mathfrak{A}} \subseteq A$, for every $p \in \mathbf{P}$. The set $A$ is called the domain of $\mathfrak{A}$. Given a structure $\mathfrak{A}$, we extend the map $p \mapsto p^{\mathfrak{A}}$ to all literals by setting $\bar{p}^{\mathfrak{A}}=A \backslash p^{\mathfrak{A}}$. We define the truth-relation $\models$ between structures and $\mathcal{N}^{\dagger}$-formulas by declaring

$$
\begin{aligned}
& \mathfrak{A} \models(\leq C)[l, m] \text { iff }\left|l^{\mathfrak{A}} \cap m^{\mathfrak{A}}\right| \leq C \\
& \mathfrak{A} \models(>C)[l, m] \text { iff }\left|l^{\mathfrak{A}} \cap m^{\mathfrak{A}}\right|>C .
\end{aligned}
$$

Note that these truth-conditions are symmetric in the literals $l$ and $m$. Accordingly, we henceforth identify formulas differing only with respect to the order of their literals, silently performing any transpositions required.

These semantics justify the following English glosses for $\mathcal{N}$-formulas, where $p$ and $q$ are English count nouns (and hence also elements of $\mathbf{P}$ ):

$$
\begin{array}{llll}
(\leq C)[p, q] & \text { At most } C p \text { are } q & (\leq C)[p, \bar{q}] & \text { At most } C p \text { are not } q \\
(>C)[p, q] & \text { At least } C+1 p \text { are } q & (>C)[p, \bar{q}] & \text { At least } C+1 p \text { are not } q .
\end{array}
$$


We also provide pseudo-English glosses for $\mathcal{N}^{\dagger}$-formulas in a similar way, except that 'negated' subjects are required when both literals are negative:

$$
\begin{array}{ll}
(\leq C)[\bar{p}, \bar{q}] & \text { At most } C \text { non- } p \text { are not } q \\
(>C)[\bar{p}, \bar{q}] & \text { At least } C+1 \text { non- } p \text { are not } q .
\end{array}
$$

The use of $\leq$ and $>$ (rather than the $\leq$ and $\geq$ employed in Section 1) simplifies various technical details in the ensuing presentation. Nothing of substance hinges on this decision, however; the results obtained below would not be materially altered by expanding our languages to include formulas of the form $(\geq 0)[l, m]$.

If $\Theta$ is a set of formulas, we write $\mathfrak{A} \models \Theta$ if, for all $\theta \in \Theta, \mathfrak{A} \models \theta$. A formula $\theta$ is satisfiable if there exists $\mathfrak{A}$ such that $\mathfrak{A} \models \theta$; a set of formulas $\Theta$ is satisfiable if there exists $\mathfrak{A}$ such that $\mathfrak{A} \models \Theta$. If, for all structures $\mathfrak{A}, \mathfrak{A} \models \Theta$ implies $\mathfrak{A} \models \theta$, we say that $\Theta$ entails $\theta$, and write $\Theta \models \theta$. We take it as uncontroversial that $\Theta \models \theta$ constitutes a rational reconstruction of the pre-theoretic judgment that a conclusion $\theta$ may be validly inferred from premises $\Theta$. For example, the valid argument (1) corresponds to the entailment

$$
\begin{aligned}
\{(>12)[\operatorname{artst}, \mathrm{bkpr}],(\leq 3)[\mathrm{bkpr}, \overline{\mathrm{crpntr}}] \\
(\leq 1)[\mathrm{crpntr}, \overline{\mathrm{dntst}}]\} \models(>8)[\text { artst, dntst }] .
\end{aligned}
$$

No formula of the form $(>C)[l, \bar{l}]$ is satisfiable: that is, for all $\mathfrak{A}$, $\mathfrak{A} \not \models(>C)[l, \bar{l}]$. We refer to any such formula as an absurdity; and we use the (possibly decorated) symbol $\perp$ to denote, ambiguously, any absurdity. Note that all absurdities are actually $\mathcal{N}$-formulas.

If $\theta$ is an $\mathcal{N}^{\dagger}$-formula, we define the $\mathcal{N}^{\dagger}$-formula $\bar{\theta}$ to be the result of exchanging the symbols $\leq$ and $>$ in $\theta$. That is:

$$
\bar{\theta}= \begin{cases}(>C)[l, m] & \text { if } \theta=(\leq C)[l, m] \\ (\leq C)[l, m] & \text { if } \theta=(>C)[l, m] .\end{cases}
$$

It is easy to see that, for any structure $\mathfrak{A}, \mathfrak{A} \models \theta$ if and only if $\mathfrak{A} \forall \bar{\theta}$. Moreover, for any $\mathcal{N}^{\dagger}$-formula $\theta$, we have $\overline{\bar{\theta}}=\theta$, and if $\theta$ is an $\mathcal{N}$-formula, then so is $\bar{\theta}$. Informally, we may think of $\bar{\theta}$ as the negation of $\theta$. Thus, the languages $\mathcal{N}^{\dagger}$ and $\mathcal{N}$ are, in essence, closed under negation.

The satisfiability problem for $\mathcal{N}^{\dagger}$ is the following problem: given a finite set of $\mathcal{N}^{\dagger}$-formulas $\Theta$, determine whether $\Theta$ is satisfiable. The validity problem for $\mathcal{N}^{\dagger}$ is the following problem: given a finite set of $\mathcal{N}^{\dagger}$-formulas $\Theta$ and an $\mathcal{N}^{\dagger}$-formula $\theta$, determine whether $\Theta \models \theta$. The satisfiability and validity problems for $\mathcal{N}$ are defined analogously. Since $\mathcal{N}^{\dagger}$ and $\mathcal{N}$ are, in effect, closed under negation, satisfiability and validity are dual notions in the usual sense. It is known [21, $14]$ that the satisfiability problems for $\mathcal{N}^{\dagger}$ and $\mathcal{N}$ are both NPTime-complete; hence the corresponding validity problems are both Co-NPTimE-complete.

\section{Proof theory for $\mathcal{N}^{\dagger}$ and $\mathcal{N}$}

This section develops a framework for formalizing systems of syllogism-like rules in $\mathcal{N}$ and $\mathcal{N}^{\dagger}$. Because we shall be deriving negative results about such systems, 
and wish these results to be as general as possible, our presentation will be in some respects rather abstract. However, we shall never stray far from the intuitions developed in Section 1.

We begin with some very general notions. Let $\mathcal{L}$ be any formal language, understood as a set of $\mathcal{L}$-formulas for which a truth-relation $\models$ is defined. By a derivation relation $($ in $\mathcal{L})$, we simply mean a subset of $\mathbb{P}(\mathcal{L}) \times \mathcal{L}$, where $\mathbb{P}(\mathcal{L})$ is the power set of $\mathcal{L}$. If $\sim$ is a derivation relation, we write $\Theta \sim \theta$ instead of $\langle\Theta, \theta\rangle \in \sim$. We call $\sim$ sound $($ for $\mathcal{L}$ ) if, for all sets of $\mathcal{L}$-formulas $\Theta$ and all $\mathcal{L}$-formulas $\theta, \Theta \sim \theta$ implies $\Theta \models \theta$. We call $\sim$ complete $($ for $\mathcal{L}$ ) if, for all sets of $\mathcal{L}$-formulas $\Theta$ and all $\mathcal{L}$-formulas $\theta, \Theta \models \theta$ implies $\Theta \sim \theta$. In this paper, we are interested in derivation relations in $\mathcal{N}^{\dagger}$ and $\mathcal{N}$ generated by finite sets of syllogism-like rules. These we now proceed to define.

A formula schema in $\mathcal{N}^{\dagger}$ is an expression of the form $(Q)[l, m]$ where $Q$ is either of the symbols $\leq$ or $>$, and $l$ and $m$ are literals. A formula schema in $\mathcal{N}$ is a formula schema in $\mathcal{N}^{\dagger}$ subject to the additional condition that at least one of $l$ and $m$ is positive. A syllogistic rule in $\mathcal{N}^{\dagger}$ (in $\mathcal{N}$ ) is a pair $(\boldsymbol{\xi}, R$ ), where, for some $k \geq 0, \boldsymbol{\xi}$ is a $(k+1)$-tuple of formula schemata in $\mathcal{N}^{\dagger}$ (respectively, $\mathcal{N}$ ), and $R$ is a $(k+1)$-ary relation over $\mathbb{N}$. A substitution is a function $f: \mathbf{P} \rightarrow \mathbf{P}$. Substitutions are applied to negative literals in the expected way: $f(\bar{p})=\overline{f(p)}$. An instance of the syllogistic rule

$$
\left(\left\langle\left(Q_{1}\right)\left[l_{1}, m_{1}\right], \ldots,\left(Q_{k}\right)\left[l_{k}, m_{k}\right],(Q)[l, m]\right\rangle, R\right)
$$

is any $(k+1)$-tuple

$$
\left\langle\left(Q_{1} C_{1}\right)\left[f\left(l_{1}\right), f\left(m_{1}\right)\right], \ldots,\left(Q_{k} C_{k}\right)\left[f\left(l_{k}\right), f\left(m_{k}\right)\right],(Q C)[f(l), f(m)]\right\rangle
$$

where $f$ is a substitution and $C_{1}, \ldots, C_{k}, C$ are integers such that $\left\langle C_{1}, \ldots, C_{k}, C\right\rangle \in R$. It is easy to see that, if (4) is a syllogistic rule in $\mathcal{N}^{\dagger}$ (or in $\mathcal{N}$ ), then the elements of (5) are $\mathcal{N}^{\dagger}$-formulas (respectively, $\mathcal{N}$-formulas). The intuitive meaning of any instance $\left\langle\theta_{1}, \ldots, \theta_{k}, \theta\right\rangle$ of a syllogistic rule is that $\theta$ may be inferred from $\theta_{1}, \ldots, \theta_{k}$. Officially, no restrictions at all are placed on the relation $R$. In practice, however, $R$ will usually be defined as $\left\{\left\langle x_{1}, \ldots, x_{k+1}\right\rangle \in\right.$ $\left.\mathbb{N}^{k+1} \mid \pi\left(x_{1}, \ldots, x_{k+1}\right)\right\}$, for some (arithmetic) expression $\pi$. In that case, we may display the syllogistic rule (4) in a more readable way as:

$$
\frac{\left(Q_{1} C_{1}\right)\left[l_{1}, m_{1}\right] \quad \cdots \quad\left(Q_{k} C_{k}\right)\left[l_{k}, m_{k}\right]}{(Q C)[l, m]}\left(\pi\left(C_{1}, \ldots, C_{k}, C\right)\right) .
$$

A syllogistic rule is valid if, for any instance $\left\langle\theta_{1}, \ldots, \theta_{k}, \theta\right\rangle$ of that rule, we have $\left\{\theta_{1}, \ldots, \theta_{k}\right\} \models \theta$ - that is to say, if all the inference steps it licenses are entailments. 
Some examples will help to motivate the rather austere definitions just given. Consider the following syllogistic rules, displayed in the style of (6):

$$
\begin{aligned}
& \frac{(\leq C)[l, \bar{m}] \quad(\leq D)[m, n]}{(\leq E)[l, n]}(E \geq C+D) \\
& \frac{(\leq C)[m, \bar{n}] \quad(>D)[l, m]}{(>E)[l, n]}(0 \leq E \leq D-C) .
\end{aligned}
$$

These syllogistic rules are easily seen to be valid. We encountered (8), in a slightly different guise (and with all literals positive), as Rule (2) in Section 1.

If $\mathrm{X}$ is a set of syllogistic rules in $\mathcal{N}^{\dagger}$, we define the relation of direct derivation relative to $\mathrm{X}$, denoted $\vdash_{\mathrm{X}}$, to be the smallest subset of $\mathbb{P}\left(\mathcal{N}^{\dagger}\right) \times \mathcal{N}^{\dagger}$ satisfying the following conditions:

1. if $\theta \in \Theta$, then $\Theta \vdash_{\mathbf{X}} \theta$;

2. if $\left\langle\theta_{1}, \ldots, \theta_{k}, \theta\right\rangle$ is an instance of some syllogistic rule in $\mathbf{X}$, and $\Theta \vdash_{\mathbf{X}} \theta_{i}$ for all $i(1 \leq i \leq k)$, then $\Theta \vdash_{\mathrm{x}} \theta$.

Instances of the relation $\vdash_{\mathbf{x}}$ can be established by derivations in the form of finite trees in the usual way. For instance, from the premises of Argument (1), two applications of Rule (8) yield the derivation

$$
\frac{(\leq 1)[\mathrm{crpntr}, \overline{\mathrm{dntst}}] \frac{(\leq 3)[\mathrm{bkpr}, \overline{\mathrm{crpntr}}] \quad(>12)[\text { artst, bkpr] }}{(>9)[\text { artst, crpntr] }},}{(>8)[\text { artst, dntst }]}
$$

which, again, we encountered in Section (1). Thus, for any set of syllogistic rules X containing (8), we have:

$$
\begin{aligned}
\{(>12)[\text { artst, bkpr }], & (\leq 3)[\mathrm{bkpr}, \overline{\operatorname{crpntr}}] \\
& (\leq 1)[\mathrm{crpntr}, \overline{\mathrm{dntst}}]\} \vdash_{\mathrm{X}}(>8)[\text { artst, dntst] }
\end{aligned}
$$

We remark in passing that, if $\mathbf{X}$ contains both Rules (7) and (8), we have an alternative derivation showing (9):

$$
\begin{aligned}
& \underline{(\leq 3)[\mathrm{bkpr}, \overline{\mathrm{crpntr}}] \quad(\leq 1)[\mathrm{crpntr}, \overline{\mathrm{dntst}}]} \text { Rule (7) }
\end{aligned}
$$

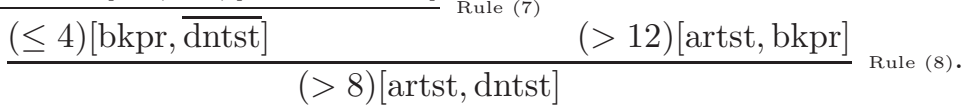

Classical treatments of the syllogistic actually recognize a slightly more liberal notion of derivation than that presented above. Suppose we have derived $\perp$ from a set of premises $\Theta \cup\{\theta\}$, where $\perp$ is some absurdity. The rule of reductio ad absurdum allows us then to infer the formula $\bar{\theta}$ (semantically: the negation of $\theta$ ) from $\Theta$ alone. Reductio is not a syllogistic rule, in the technical sense employed in this paper: for one thing it decreases the set of premises in a 
derivation - something no syllogistic rule can do. Nevertheless, it evidently preserves entailment: if $\Theta \cup\{\theta\} \models \perp$, then $\Theta \models \bar{\theta}$. For these reasons, we might wish to take account of this rule in our analysis of the numerical syllogistic.

We do so as follows. If $X$ is a set of syllogistic rules in $\mathcal{N}^{\dagger}$, we define the relation of indirect derivation relative to $\mathrm{X}$, denoted $\vdash_{\mathrm{X}}$, to be the smallest subset of $\mathbb{P}\left(\mathcal{N}^{\dagger}\right) \times \mathcal{N}^{\dagger}$ satisfying the following conditions:

1. if $\theta \in \Theta$, then $\Theta \vdash_{\mathbf{x}} \theta$;

2. if $\Theta \Vdash_{\mathbf{X}} \theta_{1}, \ldots, \Theta \Vdash_{\mathbf{X}} \theta_{k}$, and $\left\langle\theta_{1}, \ldots, \theta_{k}, \theta\right\rangle$ is an instance of some syllogistic rule in $\mathrm{X}$, then $\Theta \Vdash_{\mathrm{X}} \theta$;

3. if $\Theta \cup\{\theta\} \Vdash_{\mathbf{X}} \perp$, then $\Theta \Vdash_{\mathbf{X}} \bar{\theta}$ (the rule of reductio ad absurdum).

Instances of the indirect derivation relation $\Vdash_{x}$ may be established by constructing proof-trees similar to those for direct derivations, except that we need a little more machinery to keep track of premises. This may be done as follows. Suppose we have a derivation (direct or indirect) showing that $\Theta \cup\{\theta\} \Vdash_{x} \perp$, for some absurdity $\perp$. Let this derivation be displayed as

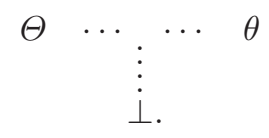

Applying Clause 3 of the definition of $\Vdash_{\mathbf{x}}$, we have $\Theta \Vdash_{\mathbf{x}} \bar{\theta}$, which we take to be established by the derivation

$$
\begin{array}{cccc}
\Theta & \cdots & \cdots & {[\theta]^{1}} \\
& \vdots & \\
& & \perp & \\
& & & \\
& & & \\
& & \text { RAA })^{1} .
\end{array}
$$

The premise $\theta$ of the original derivation no longer counts as a premise in the new derivation. As we say, the premise in question has been discharged. In displaying derivations, we enclose any discharged premise in brackets, and co-index it with the application of reductio which discharges it. Two minor complications regarding indirect derivations should be noted at this point. The first concerns the case where a derivation of $\perp$ involves multiple instances of some premise $\theta$. In that case, the rule of reductio should be understood as allowing us to discharge any number (including zero) of those occurrences. The second complication concerns the case where a derivation of $\perp$ involves no instances of some premise $\theta$. In that case, the rule of reductio should be understood as still allowing us to discharge (zero occurrences of) $\theta$. Put another way: we do not have to discharge occurrences of premises if we do not want.

If $\mathrm{X}$ is a set of syllogistic rules in $\mathcal{N}$, we define the derivation relations $\vdash_{\mathrm{X}}$ and $\Vdash_{x}$ analogously.

Thus, given a set of syllogistic rules $X$ (in either $\mathcal{N}^{\dagger}$ or $\mathcal{N}$ ), we have two derivation relations of interest: $\vdash_{\mathbf{X}}$ (direct derivation) and $\vdash_{\mathrm{X}}$ (indirect derivation), with the latter always including the former. The following are evidently 
equivalent: (i) $\vdash_{x}$ is sound; (ii) $\vdash_{x}$ is sound; (iii) every rule in $\mathrm{X}$ is valid. Moreover, if $\vdash_{\mathrm{x}}$ is complete, then, trivially, so is $\vdash_{\mathrm{x}}$.

The following questions now arise. Does there exist a finite set $X$ of syllogistic rules in $\mathcal{N}^{\dagger}$ such that the direct derivation relation $\vdash_{\mathrm{X}}$ is sound and complete? If not, does there at least exist a finite set $X$ of syllogistic rules in $\mathcal{N}^{\dagger}$ such that the indirect derivation relation $\Vdash_{X}$ is sound and complete? And is the situation any different for the smaller language $\mathcal{N}$ ? The main result of this paper is that the answer to all of these questions is no.

We close this section with a simple observation on derivations. Suppose $\Theta$ is a set of $\mathcal{N}^{\dagger}$-formulas and $\theta$ an $\mathcal{N}^{\dagger}$-formula such that $\Theta \Vdash_{\mathbf{x}} \theta$; and let $P$ be the signature of atoms occuring in $\Theta \cup\{\theta\}$. Consider any (indirect) derivation of $\theta$ from $\Theta$ (via the syllogistic rules $\mathrm{X}$ ). If that derivation involves any atoms not in $P$, we may evidently uniformly replace them by atoms in $P$, obtaining another derivation of $\theta$ from $\Theta$. The same holds for direct derivations, and also for the language $\mathcal{N}$. Thus, when considering derivations from $\Theta$ to $\theta$, we may limit ourselves entirely to the languages $\mathcal{N}^{\dagger}(P)$ or $\mathcal{N}(P)$.

\section{Main result}

Let $n$ be an integer $(n \geq 4)$, and let $P^{(n)}$ be a signature of cardinality $n+1-$ say $\left\{p_{1}, \ldots, p_{n}, q\right\}$. We denote by $\Gamma^{(n)}$ the following (infinite) set of $\mathcal{N}^{\dagger}\left(P^{(n)}\right)$ formulas, where $i, j$ range over all distinct integers in the interval $1, \ldots, n, C$ ranges over all natural numbers in the intervals indicated, and $o$ ranges over $P^{(n)}$.

1. There are exactly $n-1$ objects in the domain, all satisfying $q$ :

$$
\begin{aligned}
& (\leq C)[q, q] \quad(C \geq n-1) \quad(\leq C)[\bar{q}, \bar{q}] \quad(C \geq 0) \\
& (>C)[q, q] \quad(C \leq n-2) \text {. }
\end{aligned}
$$

2. Each $p_{i}$ is realized exactly once; and its complement is realized exactly $n-2$ times:

$$
\begin{aligned}
& (\leq C)\left[p_{i}, p_{i}\right] \quad(C \geq 1) \quad(\leq C)\left[\bar{p}_{i}, \bar{p}_{i}\right] \quad(C \geq n-2) \\
& (>0)\left[p_{i}, p_{i}\right] \quad(>C)\left[\bar{p}_{i}, \bar{p}_{i}\right] \quad(C \leq n-3) .
\end{aligned}
$$

3. All the $p_{i}$ and all the non- $p_{i}$ are $q$ :

$$
\begin{aligned}
& (\leq C)\left[p_{i}, q\right] \quad(C \geq 1) \quad(\leq C)\left[p_{i}, \bar{q}\right] \quad(C \geq 0) \\
& (>0)\left[p_{i}, q\right] \\
& (\leq C)\left[\bar{p}_{i}, q\right] \quad(C \geq n-2) \quad(\leq C)\left[\bar{p}_{i}, \bar{q}\right] \quad(C \geq 0) \\
& (>C)\left[\bar{p}_{i}, q\right] \quad(C \leq n-3) \text {. }
\end{aligned}
$$

4. No $p_{i}$ is a $p_{j}$ (remember that $\left.i \neq j\right)$ :

$$
\begin{array}{ll}
(\leq C)\left[p_{i}, p_{j}\right] & (C \geq 0) \\
(\leq C)\left[p_{i}, \bar{p}_{j}\right] & (C \geq 1) \\
(>0)\left[p_{i}, \bar{p}_{j}\right] &
\end{array}
$$

$$
\begin{array}{ll}
(\leq C)\left[\bar{p}_{i}, \bar{p}_{j}\right] & (C \geq n-3) \\
(>C)\left[\bar{p}_{i}, \bar{p}_{j}\right] & (C \leq n-4)
\end{array}
$$


5. The logical truths of $\mathcal{N}^{\dagger}\left(P^{(n)}\right)$ :

$$
(\leq C)[o, \bar{o}] \quad(C \geq 0)
$$

When considering derivations from $\Gamma^{(n)}$, we limit ourselves entirely to the language $P^{(n)}$. Where $n$ can be regarded as a constant, we omit it, and write $\Gamma$ for $\Gamma^{(n)}$.

Lemma 1. $\Gamma$ is unsatisfiable.

Proof. The formulas $(\leq n-1)[q, q],(>0)\left[p_{i}, q\right](1 \leq i \leq n)$ and $(\leq 0)\left[p_{i}, p_{j}\right]$ $(1 \leq i<j \leq n)$ together violate the pigeonhole principle.

Lemma 2. For every $\theta \in \mathcal{N}^{\dagger}\left(P^{(n)}\right)$, either $\theta \in \Gamma$ or $\bar{\theta} \in \Gamma$.

Proof. Exhaustive check.

For all $i,(1<i \leq n)$, define

$$
\begin{array}{ll}
\gamma_{i}=(\leq 0)\left[p_{1}, p_{i}\right] & \delta_{i}=(>0)\left[p_{1}, \bar{p}_{i}\right] \\
\epsilon_{i}=(>0)\left[\bar{p}_{1}, p_{i}\right] & \zeta_{i}=(\leq n-3)\left[\bar{p}_{1}, \bar{p}_{i}\right],
\end{array}
$$

so that

$$
\begin{array}{ll}
\bar{\gamma}_{i}=(>0)\left[p_{1}, p_{i}\right] & \bar{\delta}_{i}=(\leq 0)\left[p_{1}, \bar{p}_{i}\right] \\
\bar{\epsilon}_{i}=(\leq 0)\left[\bar{p}_{1}, p_{i}\right] & \bar{\zeta}_{i}=(>n-3)\left[\bar{p}_{1}, \bar{p}_{i}\right] .
\end{array}
$$

And for all $i,(1<i \leq n)$, define

$$
\Theta_{i}=\left\{\gamma_{i}, \delta_{i}, \epsilon_{i}, \zeta_{i}\right\} \quad \bar{\Theta}_{i}=\left\{\bar{\gamma}_{i}, \bar{\delta}_{i}, \bar{\epsilon}_{i}, \bar{\zeta}_{i}\right\}
$$

Note that $\Theta_{i} \subseteq \Gamma$; indeed, all the $\Theta_{i}$ are given in Clause 4 of the definition of $\Gamma$. (Remember: the order of literals in $\mathcal{N}^{\dagger}$-formulas is not significant.) In the presence of the formulas given in Clauses $1-3$ of the definition of $\Gamma$, any formula in $\Theta_{i}$ is equivalent to any other, and states that the interpretations of $p_{1}$ and $p_{i}$ are disjoint. Similarly, any formula in $\bar{\Theta}_{i}$ states that the interpretations of $p_{1}$ and $p_{i}$ coincide. It is not hard to see that any set $\left(\Gamma \backslash \Theta_{i}\right) \cup \bar{\Theta}_{i}$ is satisfiable. For let $A=\{2, \ldots, n\}$, and, for all $i(1<i \leq n)$, let $\mathfrak{A}_{i}$ be the structure with domain $A$ and interpretations

$$
q^{\mathfrak{A}_{i}}=A \quad p_{1}^{\mathfrak{A}_{i}}=\{i\} \quad p_{j}^{\mathfrak{A}_{i}}=\{j\} \quad(2 \leq j \leq n) .
$$

Thus, each $\mathfrak{A}_{i}$ distributes the interpretations of $p_{2}, \ldots, p_{n}$ disjointly over the universe $\{2, \ldots, n\}$, and makes the interpretations of $p_{1}$ and $p_{i}$ coincide.

Lemma 3. For all $i(1<i \leq n), \mathfrak{A}_{i} \models\left(\Gamma \backslash \Theta_{i}\right) \cup \bar{\Theta}_{i}$.

Proof. Routine check.

For all $i, j,(1<i<j \leq n)$, define

$$
\Delta_{i, j}^{(n)}=\Gamma^{(n)} \backslash\left(\Theta_{i} \cup \Theta_{j}\right)
$$

Again, for clarity, the superscript $(n)$ is omitted where $n$ can be regarded as a constant. Thus, $\Delta_{i, j}$ removes from $\Gamma$ the formulas stating that the interpretation of $p_{1}$ is disjoint from those of both $p_{i}$ and $p_{j}$. 
Lemma 4. Let $\theta$ be a formula of $\mathcal{N}^{\dagger}\left(P^{(n)}\right)$, and let $1<i<j \leq n$. If $\Delta_{i, j} \models \theta$, then $\theta \in \Delta_{i, j}$.

Proof. From Lemma 2, either $\theta \in \Gamma$ or $\bar{\theta} \in \Gamma$. Hence, if $\theta \notin \Delta_{i, j}$, then one of the following possibilities holds: (i) $\theta \in \Theta_{i}$; (ii) $\theta \in \Theta_{j}$; (iii) $\bar{\theta} \in \Delta_{i, j} \cup \Theta_{i}$; or (iv) $\bar{\theta} \in \Delta_{i, j} \cup \Theta_{j}$. From Lemma 3 , we see that, in cases (i) and (iv), the fact that $\mathfrak{A}_{i} \models \Delta_{i, j} \cup \bar{\Theta}_{i} \cup \Theta_{j}$ contradicts $\Delta_{i, j} \models \theta$, and that, in cases (ii) and (iii), the fact that $\mathfrak{A}_{j} \models \Delta_{i, j} \cup \bar{\Theta}_{j} \cup \Theta_{i}$ contradicts $\Delta_{i, j} \models \theta$.

Lemma 5. Let $\mathrm{X}$ be a finite set of valid syllogistic rules in $\mathcal{N}^{\dagger}$, and let $r$ be the maximum number of antecedents in any syllogistic rule of $\mathbf{X}$. If $\theta \in \mathcal{N}^{\dagger}\left(P^{(n)}\right)$, and $\Gamma^{(n)} \vdash_{\mathrm{x}} \theta$, where $n \geq r+3$, then $\theta \in \Gamma^{(n)}$.

Proof. We proceed by induction on the lengths of (direct) derivations. If a derivation of $\theta$ from $\Gamma^{(n)}$ employs no syllogistic rules, then, trivially, $\theta \in \Gamma^{(n)}$. For the inductive step, consider the last rule-instance $\left\langle\theta_{1}, \ldots, \theta_{k}, \theta\right\rangle$ in the derivation. By inductive hypothesis, $\left\{\theta_{1}, \ldots, \theta_{k}\right\} \subseteq \Gamma^{(n)}$. But because $k \leq r \leq n-3$, we in fact have, for some $i, j(1<i<j \leq n),\left\{\theta_{1}, \ldots, \theta_{k}\right\} \subseteq \Delta_{i, j}^{(n)}$. Since every rule in $\mathrm{X}$ is valid, $\Delta_{i, j}^{(n)} \models \theta$. By Lemma $4, \theta \in \Delta_{i, j}^{(n)} \subseteq \Gamma^{(n)}$. This completes the induction.

Note that, from Lemma 5, we see immediately that there is no finite set $\mathbf{X}$ of syllogistic rules for $\mathcal{N}^{\dagger}$ such that the direct derivation relation $\vdash_{\mathrm{x}}$ is sound and complete. For suppose $r$ is the maximum number of antecedents in any of the syllogistic rules in $\mathbf{X}$, and let $n \geq r+3$. If $\perp=(>0)[l, \bar{l}]$ is any absurdity, we have $\Gamma^{(n)} \models \perp$, by Lemma 1. But, by inspection, $\perp \notin \Gamma^{(n)}$. Of course, Lemma 5 does not by itself establish the incompleteness of the indirect system $\Vdash_{\mathrm{x}}$, which includes the rule of reductio. However, Lemma 2 ensures that reductio actually does no useful work in the present case, as we now proceed to show.

Theorem 1. There is no finite set $\mathbf{X}$ of syllogistic rules in $\mathcal{N}^{\dagger}$ such that $\Vdash_{\mathbf{X}}$ is sound and complete for $\mathcal{N}^{\dagger}$.

Proof. We assume otherwise and derive a contradiction. Suppose $\mathbf{X}$ is a finite set of syllogistic rules for the numerical syllogistic with $\Vdash_{x}$ sound and complete. Let $r$ be the maximum number of antecedents in any of the syllogistic rules in $\mathrm{X}$, and let $n \geq r+3$. For any absurdity $\perp=(>0)[l, \bar{l}]$, we have $\Gamma^{(n)} \models \perp$, by Lemma 1 . By the (assumed) completeness of $\Vdash_{\mathrm{x}}$, we have $\Gamma^{(n)} \Vdash_{\mathrm{x}} \perp$. Let $k$ be the smallest integer with the property that there is a derivation of some absurdity in $\Vdash_{x}$ from $\Gamma^{(n)}$ employing at most $k$ applications of the rule of reductio. Since $\Gamma^{(n)}$ contains no absurdities at all, it follows from Lemma 5 that $k>0$. Now take any such derivation employing the minimal number $k$ of applications of reductio, and consider the last such application, which, we may suppose, discharges (more than zero occurrences of) a premise $\theta$ as a result of deriving some absurdity 
$(>0)[m, \bar{m}]:$

$$
\begin{aligned}
& \begin{array}{llll}
\Gamma & \ldots & \cdots & {[\theta]^{1}}
\end{array}
\end{aligned}
$$

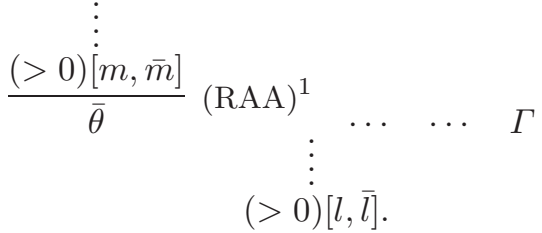

By Lemma 2, either $\theta \in \Gamma^{(n)}$ or $\bar{\theta} \in \Gamma^{(n)}$. But then either one of the smaller derivations
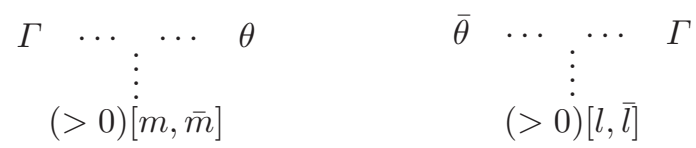

is a derivation of an absurdity from $\Gamma^{(n)}$ involving fewer than $k$ applications of reductio, which is impossible.

By restricting all formulas in the above proof to be $\mathcal{N}$-formulas, we obtain, by identical reasoning:

Theorem 2. There is no finite set $\mathrm{X}$ of syllogistic rules in $\mathcal{N}$ such that $\Vdash_{\mathbf{X}}$ is sound and complete for $\mathcal{N}$.

The details are left to the reader.

\section{Acknowledgments}

This research was supported by the EPSRC, grant number EP/F069154/1. The author would like to thank Lawrence S. Moss for his helpful comments on a draft of this paper.

\section{References}

1. Corcoran, J.: Completeness of an ancient logic. Journal of Symbolic Logic 37(4) (1972) 696-702

2. Martin, J.N.: Aristotle's natural deduction revisited. History and Philosophy of Logic 18(1) (1997) 1-15

3. Pratt-Hartmann, I., Moss, L.S.: Logics for the relational syllogistic. ArXiv preprint server, http://arxiv.org/abs/0808.0521 (2008)

4. Smiley, T.: What is a syllogism? Journal of Philosophical Logic 2 (1973) 135-154

5. de Morgan, A.: Formal Logic: or, the calculus of inference, necessary and probable. Taylor and Walton, London (1847)

6. Boole, G.: Of propositions numerically definite. Transactions of the Cambridge Philosophical Society XI(II) (1868)

7. Boole, G.: Collected Logical Works: Studies in Logic and Probability. Volume 1. Open Court, La Salle, IL (1952)

8. Jevons, W.: On a general system of numerically definite reasoning. Memoirs of the Manchester Literary and Philosophical Society (3rd Series) 4 (1871) 330-352 
9. Jevons, W.: Pure Logic and Other Minor Works. Macmillan, London (1890)

10. Grattan-Guinness, I.: The Search for Mathematical Roots, 1870-1940 : logics, set theories and the foundations of mathematics from Cantor through Russell to Gödel. Princeton University Press, Princeton, N.J. (2000)

11. Murphree, W.: The numerical syllogism and existential presupposition. Notre Dame Journal of Formal Logic 38(1) (1997) 49-64

12. Murphree, W.: Numerical term logic. Notre Dame Journal of Formal Logic 39(3) (1998) 346-362

13. Hacker, E., Parry, W.: Pure numerical Boolean syllogisms. Notre Dame Journal of Formal Logic 8(4) (1967) 321-324

14. Pratt-Hartmann, I.: On the computational complexity of the numerically definite syllogistic and related logics. Bulletin of Symbolic Logic 14(1) (2008) 1-28

15. Fitch, F.B.: Natural deduction rules for English. Philosophical Studies 24 (1973) 89-104

16. Suppes, P.: Logical inference in English: a preliminary analysis. Studia Logica 38(4) (1979) 375-391

17. Purdy, W.C.: A logic for natural language. Notre Dame Journal of Formal Logic 32(3) (1991) 409-425

18. Purdy, W.C.: Surface reasoning. Notre Dame Journal of Formal Logic 33(1) (1992) $13-36$

19. Purdy, W.C.: A variable-free logic for mass terms. Notre Dame Journal of Formal Logic 33(3) (1992) 348-358

20. Fyodorov, Y., Winter, Y., Francez, N.: Order-based inference in natural logic. Logic Journal of the IGPL 11(4) (2004) 385-416

21. Kuncak, V., Rinard, M.: Towards efficient satisfiability checking for Boolean algebra with Presburger arithmetic. In Pfenning, F., ed.: Proceedings, 21st International Conference on Automated Deduction (CADE-21). Volume 4603 of Lecture Notes in Computer Science., Berlin, Springer (2007) 215-230 